\title{
Compact and silicon-on-insulator-compatible hybrid plasmonic TE-pass polarizer
}

\author{
M. Z. Alam,* J. Stewart Aitchison and M. Mojahedi \\ Department of Electrical \& Computer Engineering, University of Toronto, Toronto, Ontario M5S3G4, Canada \\ *Corresponding author: malam@waves.utoronto.ca \\ Received July 5, 2011; revised November 4, 2011; accepted November 16, 2011; \\ posted November 17, 2011 (Doc. ID 150478); published December 24, 2011

\begin{abstract}
Hybrid plasmonic waveguides consisting of a metal plane separated from a high-index medium by a low-index spacer have recently attracted much interest. Here we show that, by suitably choosing the dimensions and material properties of the hybrid waveguide, a very compact and broadband TE-pass polarizer can be implemented. Finitedifference time-domain simulation indicates that the proposed device can provide large extinction ratio with low insertion loss for the TE mode. (C) 2011 Optical Society of America

OCIS Codes: $130.3120,240.6680,260.3910,310.6628$.
\end{abstract}

Silicon-on-insulator (SOI) technology has attracted a lot of interest as a platform for integrated optics in recent years. Because of the high dielectric contrast of silicon with air and silica, SOI waveguides can guide light in a very confined manner. The SOI platform is also compatible with complementary metal-oxide semiconductor technology and offers the potential of integration of electronics and photonics. Unfortunately, the high dielectric contrast makes SOI waveguides highly polarization dependent and this issue must be taken into account when designing any optical system. For example, the polarization state in a standard optical fiber varies randomly and connecting it directly to an SOI optical chip may disrupt the proper function of the latter. One solution to this problem is to use a polarization diversity scheme where the two polarization states are separated at the input of the SOI chip and processed separately. This results in an increased system size and complexity. Another solution that is satisfactory for many practical situations is to use a polarizer to extinguish the unwanted polarization state.

The metal clad optical polarizer consisting of a dielectric waveguide separated from a metal surface by a buffer layer is one of the most common types of integrated TEpass polarizer [1]. Such a polarizer can offer a very high extinction ratio and low insertion loss for the TE mode. However, proper operation of the metal clad polarizer requires exact phase matching between the surface plasmon and dielectric waveguide modes. Hence it is very sensitive to dimensional variations. A gap plasmon waveguide resonantly coupled to a dielectric waveguide can provide a very high extinction ratio over a broad wavelength range, but the TE insertion loss for the device is significant [2]. A shallowly etched SOI waveguide polarizer has also been proposed [3] that utilizes the polarization dependence of leakage loss to extinguish the TM mode while the TE mode is well guided. The polarizer is very simple to fabricate and provides excellent extinction ratio and low insertion loss for the TE mode. However, the polarizer is $1 \mathrm{~mm}$ long, compromising compactness, which is a key advantage of SOI photonics. A compact SOI compatible broadband TE-pass polarizer with low insertion loss for the TE mode is yet to be reported.

Recently, we proposed a hybrid plasmonic waveguide that consists of a metal plane separated from a high- index medium by a low-index spacer layer [ $[\underline{4}, \underline{5}]$. Since then a number of hybrid plasmonic waveguides have been proposed [4-7] and various practical applications have been suggested [8-10]. A hybrid waveguide that is especially suitable for SOI technology is shown in Fig. 1(a) [7]. It consists of a metal slab of size $w \times t$ separated from a silicon slab of dimension $w \times d$ (highindex medium) by a low-index spacer of dimension $w \times h$ (low-index medium). The structure is compatible with SOI fabrication technology and can be easily integrated with other SOI devices. The fundamental TM mode in the hybrid guide is concentrated in close vicinity of the metal. In contrast, the fundamental TE mode is concentrated in the high-index region. As a result, the TM mode is always more lossy for such a guide [8]. For a proper choice of waveguide dimensions, the TM mode propagating though the hybrid waveguide will suffer very high attenuation, while the TE mode will be relatively unaffected. Inserting such a hybrid waveguide

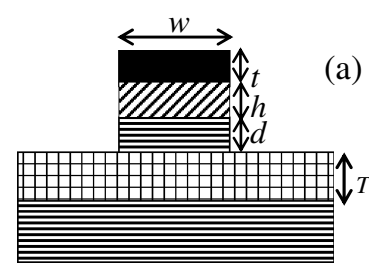

(a)
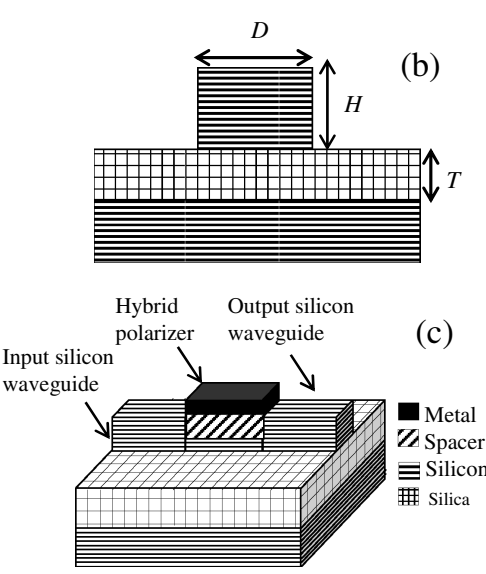

Fig. 1. Cross sections of (a) a hybrid waveguide and (b) input and output silicon waveguides, and (c) a three-dimensional schematic of the complete TE-pass polarizer. 
section between two input/output silicon waveguides [shown in Fig. 1(b)] will result in a TE-pass polarizer [shown in Fig. 1(c)].

A good TE-pass polarizer should have (a) low insertion loss for the TE mode, (b) high insertion loss for the TM mode, and (c) compact size. For ease of discussion in this work, we designate the ratio of propagation loss of the two polarizations as

$$
\eta=\frac{\text { Propagation loss of TM mode }(\mathrm{dB} / \mu \mathrm{m})}{\text { Propagation loss of TE mode }(\mathrm{dB} / \mu \mathrm{m})} .
$$

Fulfillment of Condition (a) requires good effective mode index matching for the TE mode between the input/output silicon waveguide and the hybrid waveguide section. To simultaneously satisfy Conditions (a) and (b), $\eta$ has to be large. If the propagation loss for the TM mode per unit length is large, a high TM insertion loss will be achieved over a short polarizer length, which satisfies Condition (c). The material properties and polarizer dimensions must be carefully chosen to simultaneously satisfy all three conditions.

Noble metals (gold and silver) can provide low propagation loss for plasmonic modes and are the metals of choice for most plasmonic devices. However, in the current case, we prefer large losses for the hybrid mode and a metal having a large imaginary part of permittivity in the near-IR; for example, chromium $\left(\varepsilon_{r}=-6.7+41 \times i\right.$ at $1.55 \mu \mathrm{m}$ wavelength) is a better choice. Chromium has very good adhesion to dielectric surfaces and, hence, will make the fabrication of the device less challenging. Two possible choices of spacer material are silica $\left(\varepsilon_{r}=2\right)$ and silicon nitride $\left(\varepsilon_{r}=4\right)$. Since the latter choice gives a larger propagation loss for the TM mode [8], we choose silicon nitride as the spacer material.

Since the choice of waveguide dimensions affects the two modes differently, the waveguide dimensions need to be properly chosen to achieve a large value of $\eta$. We investigated the effects of waveguide dimensions on the modal properties by using the commercial finite element code COMSOL Multiphysics. Figure 2 shows the effects of changing $h$ and $d$ for fixed waveguide width $(w=550 \mathrm{~nm})$. As shown in Fig. 2(a), propagation loss for the TM mode drops for large silicon thickness $(d)$ and spacer thickness $(h)$. The propagation loss of the TE mode drops at a faster rate with increasing $d$ and $h$ and, hence, as shown in Fig. 2(b), $\eta$ increases. Figures 2(c) and $(\mathrm{d})$ show the variations of the real part of effective mode index $N_{\text {eff }}$ for the TE and TM modes, respectively. Here, effective mode index $N_{\text {eff }}$ is defined as $N_{\text {eff }}=k / k_{0}$, where $k$ is the real part of the propagation constant of the guided mode and $k_{0}=\omega / c$ is the free-space wavenumber. A large silicon thickness $(d)$ results in a large $N_{\text {eff }}$ for the TE mode, which, in turn, results in a better match between the hybrid guide and the input/output silicon waveguides. The final device dimensions chosen are: $w=550 \mathrm{~nm}, d=120 \mathrm{~nm}, h=500 \mathrm{~nm}$, and $t=200 \mathrm{~nm}$ to simultaneously satisfy Conditions (a), (b), and (c). For these dimensions and a chromium layer on top of the spacer, propagation loss for the TM mode is $3.7 \mathrm{~dB} / \mu \mathrm{m}$ and the value of $\eta$ is 152 , whereas for gold these values become $0.2 \mathrm{~dB} / \mu \mathrm{m}$ and 143 , respectively.
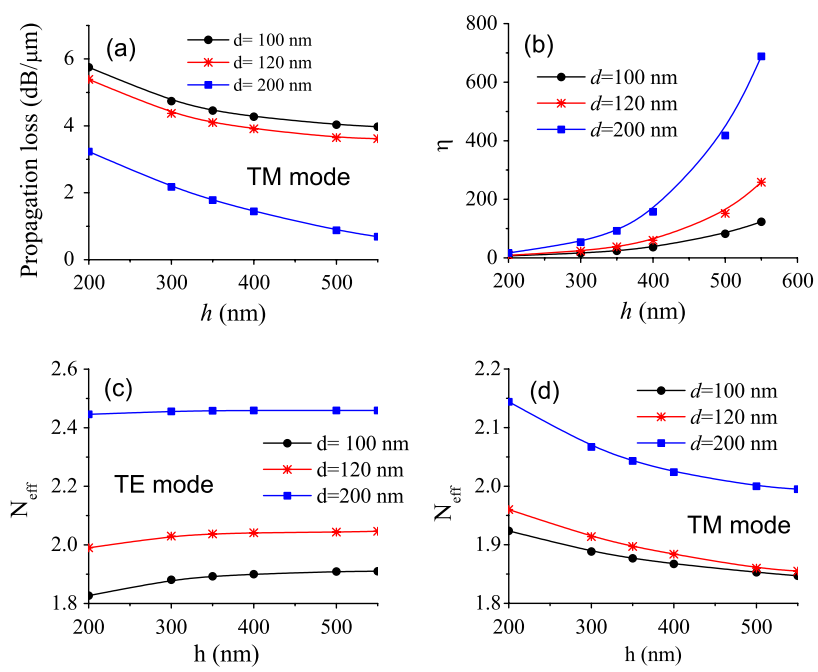

Fig. 2. (Color online) Variations of the modal characteristics with spacer and silicon thickness $(h$ and $d$ ). (a) Propagation loss of the TM mode. (b) Ratio of TM and TE loss $(\eta)$. (c) and (d) $N_{\text {eff }}$ for the TE and TM modes. Device dimensions are $w=550 \mathrm{~nm}, t=200 \mathrm{~nm}$, and $T=1.5 \mu \mathrm{m}$, and the wavelength of operation is $1.55 \mu \mathrm{m}$.

Therefore, chromium is a better choice for implementing a compact hybrid TE-pass polarizer.

The finite-difference time-domain method (FDTD) is well known for its ability to accurately predict the performance of plasmonic devices. Therefore, the commercial FDTD code Lumerical was used to investigate the performance of the final design. Because of the small skin depth of the metal, a small mesh size inside the metal is required for simulation of plasmonic devices. To achieve high accuracy without excessive demand of computational resources, we used a nonuniform mesh with a finer mesh in the metal and relatively coarse mesh in the dielectric. We carried out simulations with different mesh sizes and found that decreasing the minimum mesh size below $10 \mathrm{~nm}$ does not significantly affect the simulation results. Therefore, we used a nonuniform mesh with a minimum mesh size of $10 \mathrm{~nm}$ in our simulations. Material properties for silicon, silica, and chromium for various wavelengths are taken from [11]. The material properties of silicon nitride at telecommunication bands are not available from [11]. Different permittivity values of silicon nitride are reported in literature by different groups, but the values are all close to 4 . Therefore, for silicon nitride, we used a constant permittivity value $\left(\varepsilon_{r}=4\right)$. The permittivity of silicon nitride changes by less than $2 \%$ over the wavelength range of our investigation (1.4$1.6 \mu \mathrm{m}$ ) and neglecting the dispersion of silicon nitride should not significantly affect the results. The input/output silicon waveguide dimensions are chosen to be $D=$ $350 \mathrm{~nm}$ and $H=310 \mathrm{~nm}$. To minimize device length, no taper is used between the hybrid waveguide and silicon waveguides. The computational volume is $30 \mu \mathrm{m}$ long, $4 \mu \mathrm{m}$ wide in the lateral direction, and $3.5 \mu \mathrm{m}$ wide in the vertical direction, and is terminated with perfectly matched layers. The simulations were carried out on multiple processors in parallel on the high-performance computational facility West-grid.

Figure 3 shows the insertion loss spectrum for the TE and TM modes of a $17-\mu \mathrm{m}$-long polarizer. The hybrid 

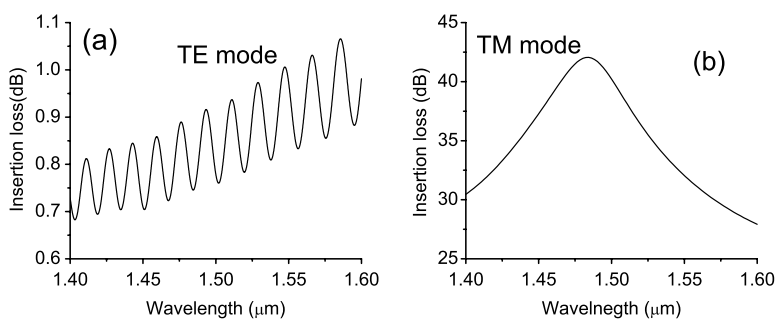

Fig. 3. Variations of insertion loss with wavelength for a 17$\mu$ m-long polarizer for the final design. (a) TE mode. (b) TM mode.

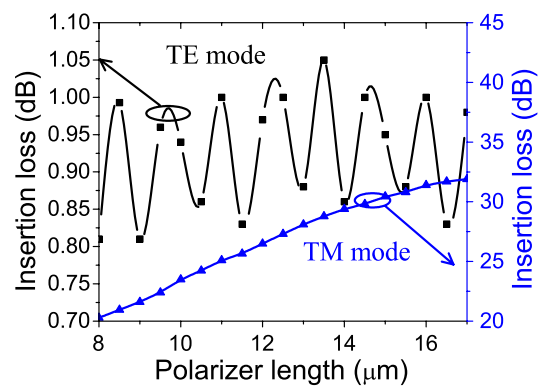

Fig. 4. (Color online) Variations of TE and TM insertion losses with polarizer length for the final design.

waveguide placed between two silicon waveguides act as a Fabry-Perot cavity, as evident from the oscillations in the TE transmission spectrum [Fig. 3(a)]. The large propagation loss diminishes the Fabry-Perot effect in case of TM mode and the TM transmission spectrum [Fig. 3(b)] exhibits no oscillation. For a given extinction ratio, the device is more compact than previously reported metal clad polarizers $[\underline{1}, \underline{2}, \underline{12}]$.To the best of our knowledge, the most compact broadband TE-pass polarizer for SOI reported so far has a length of $120 \mu \mathrm{m}$ [2]. Simulation results for the polarizer reported in [2] predicted an insertion loss of more than $30 \mathrm{~dB}$ for the TM mode and 2 to $4 \mathrm{~dB}$ insertion loss for the TE mode over a bandwidth of $350 \mathrm{~nm}$. In contrast, as shown in Figs. 3(a) and (b), our proposed TE-pass polarizer suffers much lower insertion loss for the TE mode (approximately $1 \mathrm{~dB}$ ) and can achieve greater than $30 \mathrm{~dB}$ insertion loss for the TM mode for more than $160 \mathrm{~nm}$ bandwidth. Although the bandwidth is lower than that reported in [2], it covers the entire $S$ and $C$ bands and the device length is only $14.2 \%$ of that reported in [2].

Figure 4 shows the insertion losses for the TE and TM modes for various polarizer lengths at $1.55 \mu \mathrm{m}$ wavelength. A large extinction ratio (difference between the insertion losses for the TE and TM modes) can be achieved for a very short polarizer length. For example, an 8- $\mu \mathrm{m}$-long polarizer provides an extinction ratio of approximately $20 \mathrm{~dB}$. The TE insertion loss undergoes sinusoidal variation as a function of polarizer length due to the Fabry-Perot effect. The insertion loss is slightly higher for a polarizer length of around $14 \mu \mathrm{m}$. For the optimized design, most power for the TE mode propagates through the hybrid waveguide from the input to output silicon waveguide. However, part of the power leaks into the buried oxide layer, reflects back at the buried oxide/ silicon substrate interface, and then couples to the output guide. The amount of this coupling and, hence, insertion loss, is maximum for a certain polarizer length. This phenomenon is well known for waveguide polarizers and is explained in more detail in [13].

In conclusion, we have proposed a compact, SOI compatible hybrid TE-pass polarizer that can be directly integrated with silicon nanowires on SOI wafer without using any taper. The extinction ratio is very high and the insertion loss for the TE mode is comparable to or less than other recently reported SOI compatible TE-pass polarizers having much larger footprints.

\section{References}

1. V. K. Sharma, A. Kumar, and A. Kapoor, Opt. Commun., 284, 1815 (2011).

2. I. Avrutsky, IEEE J. Sel. Top. Quantum Electron. 14, 1509 (2008).

3. D. Dai, Z. Wang, N. Julian, and J. E. Bowers, Opt. Express 18, 27404 (2010).

4. M. Z. Alam, J. Meier, J. S. Aitchison, and M. Mojahedi, in Conference on Lasers and Electro-Optics/Quantum Electronics and Laser Science Conference and Photonic Applications Systems Technologies, OSA Technical Digest Series (Optical Society of America, 2007), paper JThD112.

5. M. Z. Alam, J. Meier, J. S. Aitchison, and M. Mojahedi, Opt. Express 18, 12971 (2010).

6. R. F. Oulton, V. J. Sorger, D. A. Genov, D. F. P. Pile, and X. Zhang, Nat. Photon. 2, 496 (2008).

7. D. Dai and S. He, Opt. Express 17, 16646 (2009).

8. M. Z. Alam, J. S. Aitchison, and M. Mojahedi, Appl. Opt. 50, 2294 (2011).

9. R. F. Oulton, V. J. Sorger, T. Zentgraf, R-M. Ma, Christopher Gladden, Lun Dai, G. Bartal, and X. Zhang, Nature 461, 629 (2009).

10. M. Z. Alam, F. Bahrami, J. S. Aitchison, and M. Mojahedi, in CLEO 2011-Laser Applications to Photonic Applications, OSA Technical Digest (CD) (Optical Society of America, 2011), paper JWA111.

11. E. D. Palik, Handbook of Optical Constants of Solids (Academic, 1985).

12. G. Li and A. Xu, J. Lightwave Technol. 26, 1234 (2008).

13. Q. Wang and S.-T. Ho, IEEE Photon. J. 2, 49 (2010). 